\title{
Diffractive production of $\pi^{-} \pi^{-} \pi^{+}$and $\pi^{-} \pi^{0} \pi^{0}$ systems at VES.
}

\author{
Dmitry Ryabchikov*† \\ Institute for High Energy Physics, Technical University of Munich \\ E-mail: ryabchikecern.ch

\section{Igor Kachaev} \\ Institute for High Energy Physics \\ E-mail: Igor.Katchaev@ihep.ru
}

The VES experiment has collected high statistics of exclusive reactions with three pions in the final state: $\pi^{-} \pi^{-} \pi^{+}$(about $43 \cdot 10^{6}$ events) and $\pi^{-} \pi^{0} \pi^{0}$ (about $20 \cdot 10^{6}$ events). The $3 \pi$ systems are produced by impinging of $28.9 \mathrm{GeV} \pi^{-}$beam on the beryllium target. The dominant production mechanism is the Pomeron exchange between incoming beam pion and the target. At the very low momentum transfer squared $t^{\prime}$ the data clearly shows coherent diffractive production on the whole nucleus which is replaced by incoherent process on the individual nucleons at higher $0.05<t^{\prime}<1 \mathrm{GeV}^{2}$. The mass-independent partial wave analysis is performed dividing data into the bins of $m(3 \pi)$ and $t^{\prime}$. The comparison of results with two models of PWA: using formalism of unlimited-rank density matrix and, using rank $=1$ is presented. The predicted isospin relations (assuming $I=1$ of $3 \pi$ systems) and observed ones for intensities in $\pi^{-} \pi^{-} \pi^{+}$and $\pi^{-} \pi^{0} \pi^{0}$ are shown.

XVII International Conference on Hadron Spectroscopy and Structure

25-29 September, 2017

University of Salamanca, Salamanca, Spain

\footnotetext{
${ }^{*}$ Speaker.

${ }^{\dagger}$ For the VES group.
} 


\section{The reaction. PWA framework. Isospin relations.}

We present the preliminary analysis of two reactions: $\pi^{-} B e \rightarrow \pi^{-} \pi^{-} \pi^{+} B e$ (about $43 \cdot 10^{6}$ events) and $\pi^{-} B e \rightarrow \pi^{-} \pi^{0} \pi^{0} B e$ (about $20 \cdot 10^{6}$ events), with $\pi^{-}$beam of $28.9 \mathrm{GeV}$ and beryllium target. The data were taken with upgraded VES detector, using secondary negatively charged beam from the U70 proton synchrotron in Protvino. The previous analysis of $3 \pi$ states at upgraded VES can be found in [2],[3],[4],[5],[6].

The partial-wave analysis of the reaction $\pi^{-} N \rightarrow 3 \pi N$ assumes that $\pi^{-}$beam particle is excited into $3 \pi$ system by $t$-channel reggeon exchange so that nucleus or nucleon $N$ stays intact and gets very small momentum transfer $t^{\prime}=|t|-|t|_{\min } \sim q_{t}^{2}$ ( $q_{t}$ is recoil 3-momenta perpendicular to the incoming beam direction). The $3 \pi$ final states are described by $J^{P C} M^{\varepsilon}[$ isobar $] L$ quantum numbers where $J$ is total spin of $3 \pi$ system, $M>0$ is spin projection in the Gottfried-Jackson frame, $\varepsilon= \pm 1$ is reflectivity. The dipion intermediate state is called [isobar] having definite spin $S$ and isospin $I_{2 \pi}$, $L$ is relative orbital angular momentum between isobar and batchelor pion. The included isobars are $\rho(770), \rho_{3}(1690)$ with isospin $I_{2 \pi}=1$ and $f_{0}(600), f_{0}(980), f_{0}(1500), f_{2}(1270)$ with $I_{2 \pi}=0$. The complex functions describing process $J^{P C} M^{\varepsilon} \rightarrow([\text { isobar }] \pi)_{L}$ are called the decay amplitudes $\psi_{i}^{\varepsilon}(\tau)$ and are the functions of 3-body phase space variables $\tau$. The mass-independent PWA model has probability density $P(\tau)=\sum_{\varepsilon} \sum_{i, j} \rho_{i j} \psi_{i}^{\varepsilon}(\tau) \psi_{j}^{\mathcal{\varepsilon} *}(\tau)$ where $\rho_{i j}$ is spin-density matrix. It contains information on amplitudes intensities and their relative phases. Two different PWA frameworks were applied: using rank=1 of spin-density matrix and using unlimited-rank spin-density matrix. In the last case the eigenvector corresponding to the largest eigenvalue (LEV-eigenvector) can be extracted [1].

In the isobar model, relations are expected for amplitudes between $\pi^{-} \pi^{-} \pi^{+}$and $\pi^{-} \pi^{0} \pi^{0}$. In case of $I_{2 \pi}=1$ isobars the resulting $3 \pi$ isospin value can be $I_{3 \pi}=1$ and $I_{3 \pi}=2$. Assuming $I_{3 \pi}=1$ (which is true if Pomeron exchange dominates) isospin couplings connect $\rho^{0} \pi^{-}$and $\rho^{-} \pi^{0}$ states to have same intensities (taking into account same structure of Bose-symmetrization). In case of $I_{2 \pi}=0$ isobars, $\pi^{-} \pi^{0} \pi^{0}$ and $\pi^{-} \pi^{-} \pi^{+}$have $I_{3 \pi}=1$ and are connected just by isospin relation between $f \rightarrow \pi^{0} \pi^{0}$ and $f \rightarrow \pi^{+} \pi^{-}$which gives $0.5: 1$ for their relative intensities in case of pure two-body decay. However, full 3-body amplitudes with $f \pi$ intermediate channel have different structure after Bose-symmetrization. Interference of two $f \pi^{-}$terms on $\pi^{-} \pi^{-} \pi^{+}$Dalitzplot (which is absent in case of $\pi^{-} \pi^{0} \pi^{0}$ ) can break "naive" 0.5:1 ratio. The exact ratios can be calculated directly from the phase-space integrals of squared decay amplitudes, used in PWA.

\section{Results for different $J^{P C} M^{\varepsilon}$}

The high statistics of both reactions allows to perform PWA dividing the data into $20 \mathrm{MeV}$ $m(3 \pi)$-bins and separately in six $t^{\prime}$-bins 0-0.015-0.033-0.060-0.060-0.090-0.200-1 GeV.

The $2^{++} 1^{+} \rho(770) \pi D$ wave is strongly dominated by the $a_{2}(1320)$ meson signal. Its intensities as functions of $m(3 \pi)$ are shown on fig. 1 separately in six $\mathrm{t}^{\prime}$ bins for $\pi^{-} \pi^{-} \pi^{+}$system. The unlimited-rank density-matrix intensity (red) matches with intensity based on the largest eigenvalue (green) and with rank=1 model intensity (blue). However, at lowest $t^{\prime}$ bins where $a_{2}$-signal is relatively small, the unlimited-rank density matrix intensity without extracting LEV shows a leakage - which motivates us to exract largest eigenvalue from density matrix. On the other hand, dominance of $a_{2}(1320)$ signal in the LEV-eigenvector at very different values of $t^{\prime}$ demonstrates 

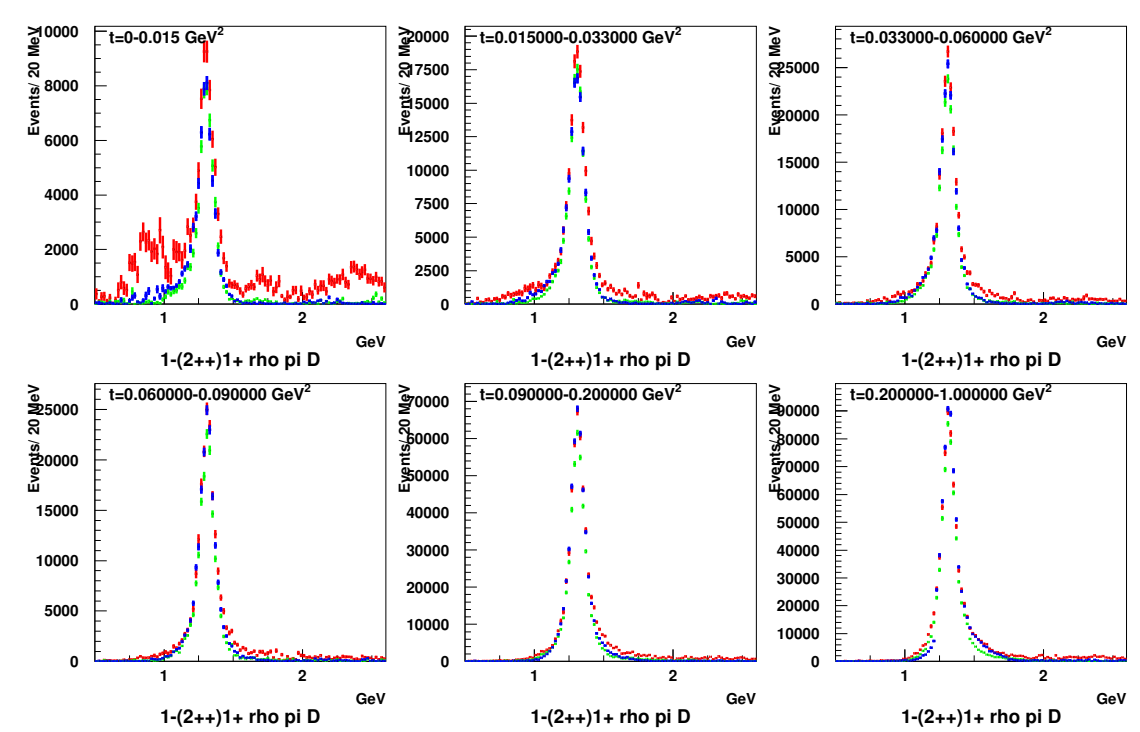

Figure 1: Intensities of $2^{++} 1^{+} \rho \pi D$ in $6 \mathrm{t}^{\prime}$-bins. Red color shows unlimited-rank density matrix model, green - the largest eigenvalue and blue shows rank $=1$ model.

high coherence between dominant amplitudes both for coherent diffraction on nuclei and also for incoherent processes on single nucleons.

The PWA intensities of $2^{++}$for $\pi^{-} \pi^{-} \pi^{+}$(in red here and later) and $\pi^{-} \pi^{0} \pi^{0}$ (in blue), their ratios (in green) and calculated ratios from PWA normalization integrals (in purple) are shown in fig. 2, for the $\mathrm{r}=1$ method and for the LEV-eigenvector. No additional scaling factor between two systems is applied. The plots show rather good agreement between calculated and measured ratious in the $a_{2}(1320)$ peak region.
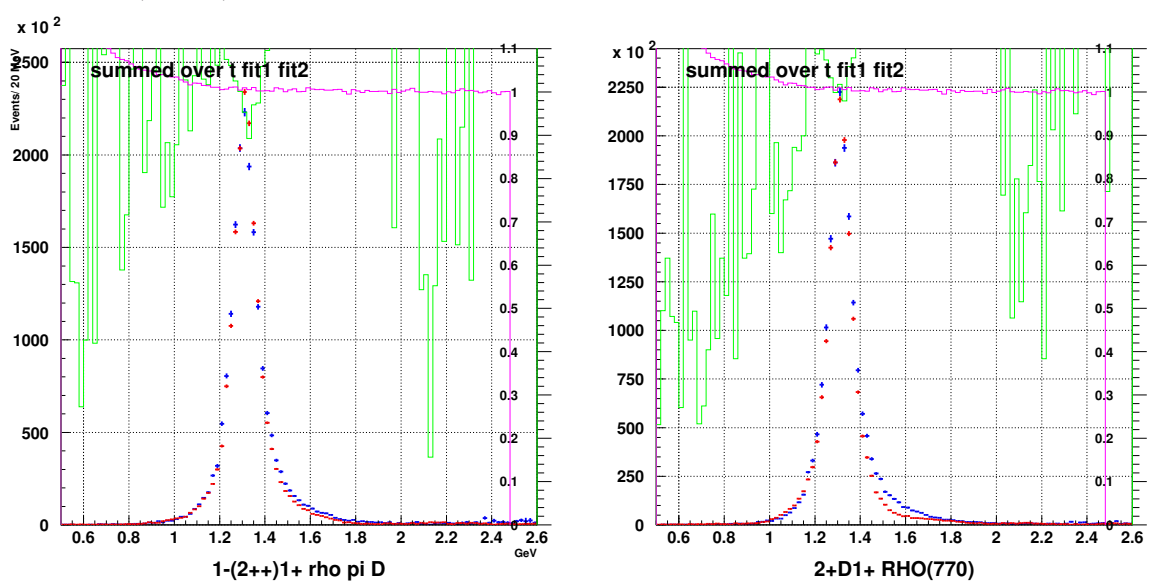

Figure 2: Intensities summed over $\mathrm{t}^{\prime}$ of $2^{++} 1^{+} \rho \pi D$ for $\pi^{-} \pi^{-} \pi^{+}$(red) and $\pi^{-} \pi^{0} \pi^{0}$ (blue), using rank=1 (left) and LEV-eigenvector (right). No any scaling factor between different methods is applied here and further.

Figure 3 shows intensities of $1^{++} 0^{+} \rho \pi S$, which dominates in $3 \pi$ states. The peak in $\pi^{-} \pi^{0} \pi^{0}$ is significantly lower, similarly in two fit models. In contrast to $2^{++}$, the $1^{++} \rho \pi S$ is not dominated by a single resonance. Visible violation of isospin relation could have several reasons. Reason 
could be inadequite parameterization of badly known $f_{0}(600)$ isobar which contributes $10-20 \%$ to $1^{++}$states. Also the admixture of $K^{+} K^{-} \pi^{-}$which amounts $\sim 10 \%$ of $\pi^{-} \pi^{-} \pi^{+}$sample can influence the experimental ratio.
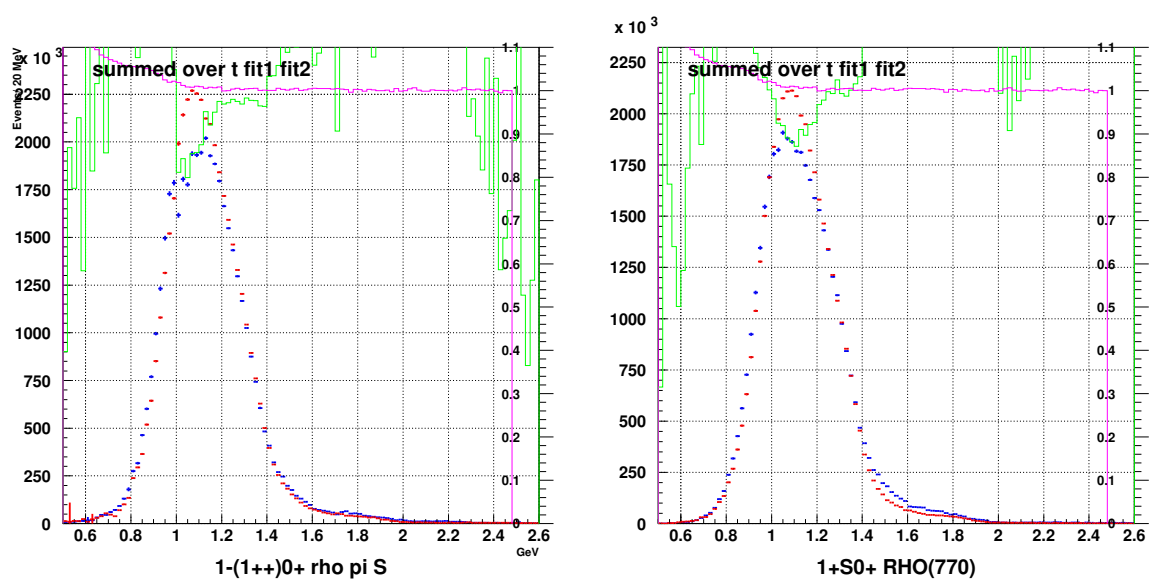

Figure 3: Intensities summed over $\mathrm{t}^{\prime}$ of $1^{++} 0^{+} \rho \pi S$ for $\pi^{-} \pi^{-} \pi^{+}$(red) and $\pi^{-} \pi^{0} \pi^{0}$ (blue), using rank=1 (left) and LEV-eigenvector (right)

The small signal in $1^{++} 0^{+} f_{0}(980) \pi P$, see fig. 4 forms rather narrow peak at about 1.45 $\mathrm{GeV}$, and was claimed as $a_{1}(1420)$ meson [7]. Here, the method of unlimited-rank density matrix demonstrates significantly lower intensity than rank $=1$ method in both $3 \pi$-systems. The observed isospin ratio is also somewhat smaller for $\pi^{-} \pi^{0} \pi^{0}$ than predicted value. To clarify the reason of those deviations, further studies are needed. Particularly, better knowledge of $f_{0}(600)$ and $f_{0}(980)$ amplitudes is needed, which are known to heavily interfere in $a_{1}(1420)$ region.
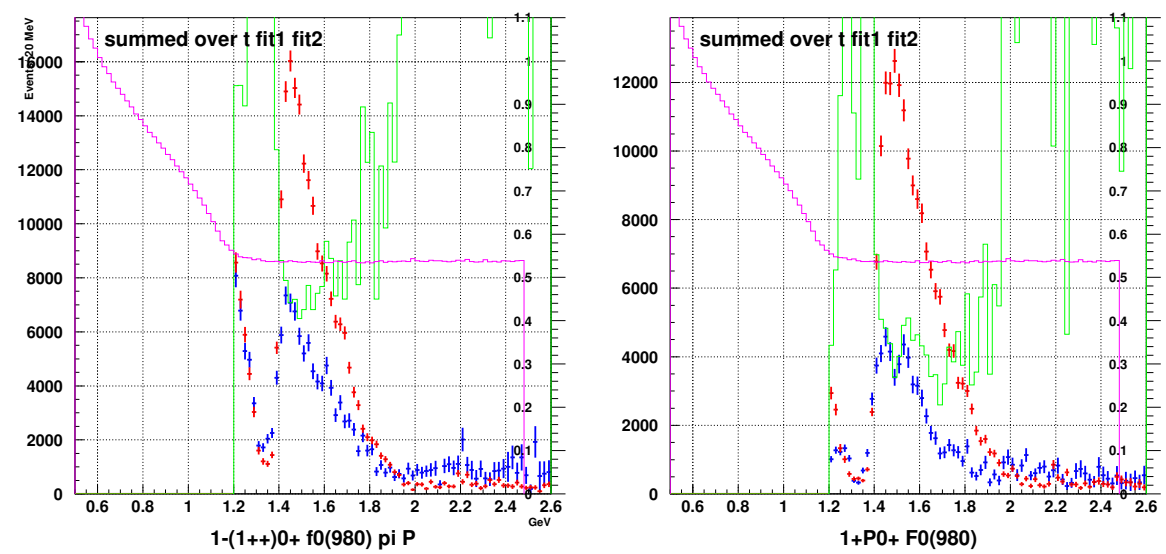

Figure 4: Intensities summed over $\mathrm{t}^{\prime}$ of $1^{++} 0^{+} f_{0}(980) \pi P$ for $\pi^{-} \pi^{-} \pi^{+}$(red) and $\pi^{-} \pi^{0} \pi^{0}$ (blue), using rank=1 (left) and LEV-eigenvector (right)

Another partial wave with $f_{0}(980)$ isobar is $0^{-+} 0^{+} f_{0}(980) \pi S$, see fig. 5. It shows clear signal of $\pi(1800)$, having low-mass background shoulder. This is an example when two PWA models give very similar results - concerning heights of the peaks, and also isospin relations at the resonant peak. 

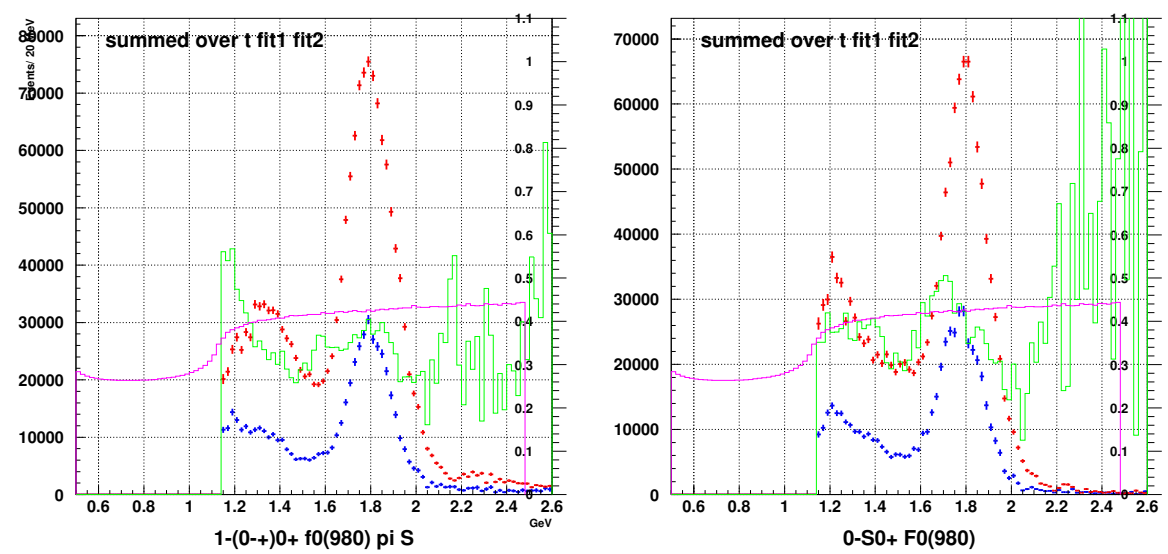

Figure 5: Intensities summed over $\mathrm{t}^{\prime}$ of $0^{-+} 0^{+} f_{0}(980) \pi S$ for $\pi^{-} \pi^{-} \pi^{+}$(red) and $\pi^{-} \pi^{0} \pi^{0}$ (blue), using rank=1 (left) and LEV-eigenvector (right)

Finally, two higher spin partial waves: $3^{++} 0^{+} \rho_{3}(1690) \pi S$ (see fig. 6) and $4^{++} 1^{+} \rho(770) \pi G$ (see fig. 7) are compared. For the first time isospin relation is studied for amplitude containing $\rho_{3}(1690)$ as an isobar. Two PWA models give very similar peak height in $\pi^{-} \pi^{-} \pi^{+}$. The corresponding peak in $\pi^{-} \pi^{0} \pi^{0}$ is excessive, but it clearly sits on low-mass background, better seen in fig. 6 right plot. $4^{++} 1^{+}$looks very clean and demonstrates reasonable isospin symmetry at the peak, however its amount is significantly lower for unlimited rank method.
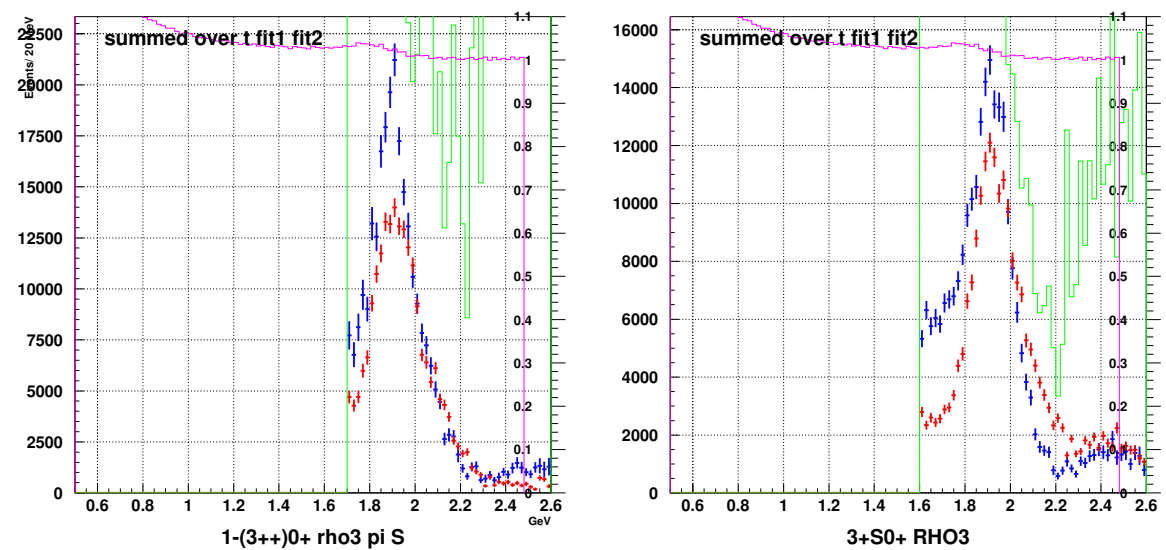

Figure 6: Intensities summed over $\mathrm{t}^{\prime}$ of $3^{++} 0^{+} \rho_{3}(1690) \pi S$ for $\pi^{-} \pi^{-} \pi^{+}$(red) and $\pi^{-} \pi^{0} \pi^{0}$ (blue), using rank=1 (left) and LEV-eigenvector(right)

\section{Conclusions and outlook}

We present for the first time the comparison of PWA of two reactions $\pi^{-} B e \rightarrow \pi^{-} \pi^{-} \pi^{+} B e$ and $\pi^{-} B e \rightarrow \pi^{-} \pi^{0} \pi^{0} B e$ using two different PWA frameworks rank=1 model for the spin-density matrix and unlimited-rank density matrix (with further extracting LEV-eigenvector). The isospin relations between amplitudes of two reactions are presented as ratious of intensities and compared with calculated values within isobar model. The results using rank=1 method are mostly compatible with unlimited-rank method and extracting of largest-eigenvalue amplitudes, however the reduction of intensities at the peaks of resonances is observed when using the second method. The intensities 

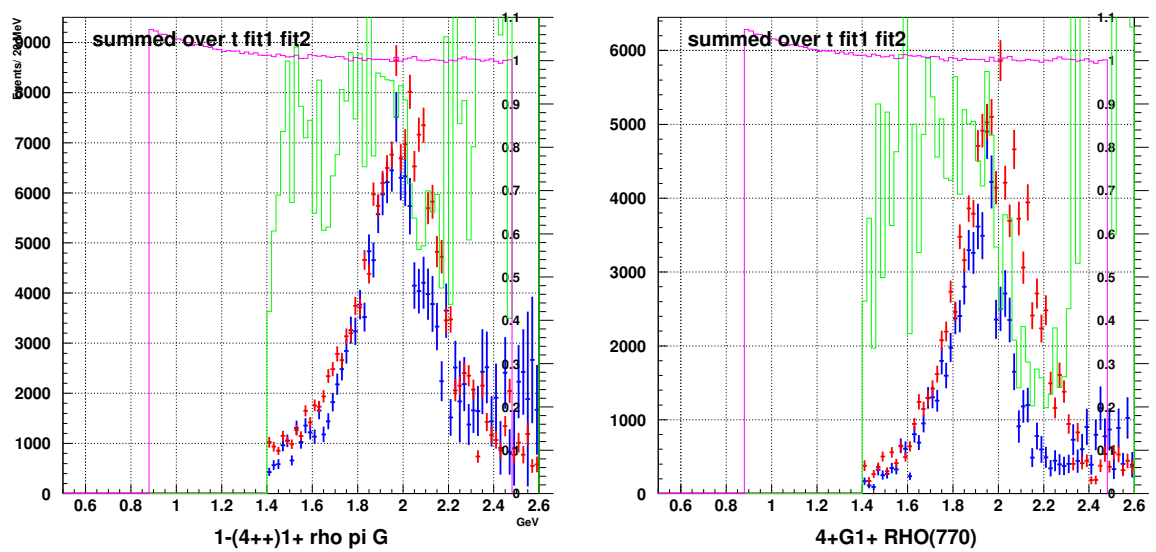

Figure 7: Intensities summed over $\mathrm{t}^{\prime}$ of $4^{++} 1^{+} \rho(770) \pi G$ for $\pi^{-} \pi^{-} \pi^{+}$(red) and $\pi^{-} \pi^{0} \pi^{0}$ (blue), using rank=1 (left) and LEV-eigenvector (right)

obtained from the unlimited-rank density matrix (without extraction of LEV-eigenvector) contain leakages - especially in case when a given intensity is relatively small. Results in different $J^{P C}$ sectors are compared in detail: $J^{P C}=0^{-+}, 1^{++}, 2^{++}, 3^{++}$and $4^{++}$Small but interesting signals are presented such as $1^{++} 0^{+} f_{0}(980) \pi$ and $3^{++} 0^{+} \rho_{3}(1690) \pi$. They demonstrate similar results using two PWA methods and reasonable isospin relations.

\section{Acknowledgments}

We got supported by Transregional Research Centre TR110 and RFBR grant 16-02-00737.

\section{References}

[1] I. Kachaev PWA tools and methods developed by VES group Talk at ATHOS-2013, http://www.athos2013.de

[2] I. Kachaev, D. Ryabchikov PWA with full rank density matrix of the $\pi^{+} \pi^{-} \pi^{-}$and $\pi^{-} \pi^{0} \pi^{0}$ systems at VES setup EPJ Web Conf. 130 (2016) 04003

[3] D. Ryabchikov et al. Analysis of the reaction $\pi^{-} B e \rightarrow \pi^{-} \pi^{0} \pi^{0}$ Be at VES AIP Conf.Proc. 1701 (2016) 040020

[4] I. Kachaev et al. Study of the reaction $\pi^{-} A \rightarrow \pi^{+} \pi^{-} \pi^{-} A$ at large statistics with VES setup Phys.Atom.Nucl. 78 (2015) no.13, 1474-1477

[5] I. Kachaev et al. Partial Wave Analyses of the $\pi^{+} \pi^{-} \pi^{-}$System at upgraded VES Setup Conference: C14-08-24, p.185-189.

[6] Yu. Khokhlov et al. Partial wave analyses of the $\pi^{-} \pi^{0} \pi^{0}$ and $\pi^{-} \pi^{0}$ systems with VES setup in proceedings of HADRON2013 conference PoS (Hadron2013) 088 (2013).

[7] C. Adolph et al. Observation of a New Narrow Axial-Vector Meson a1(1420) Phys.Rev.Lett. 115 (2015) no.8, 082001. 